\title{
Bone disease in primary hypercalciuria
}

\author{
Stefania Sella \\ Catia Cattelan \\ Giuseppe Realdi \\ Sandro Giannini
}

Department of Medical and Surgical Sciences, University of Padua, Padua, Italy

Address for correspondence:

Stefania Sella, M.D.

Department of Medical and Surgical Sciences

Clinica Medica 1, Padova University Hospital

Via Giustiniani 2, 35128 Padova, Italy

Ph. +390498212169

Fax +390498212151

E-mail: stefania.sella@unipd.it

\section{Summary}

Primary Hypercalciuria (PH) is very often accompanied with some degrees of bone demineralization. The most frequent clinical condition in which this association has been observed is calcium nephrolithiasis. In patients affected by this disorder bone density is very frequently low and increased susceptibility to fragility fractures is reported. The very poor definition of this bone disease from a histomorphometric point of view is a crucial aspect. At present, the most common finding seems to be a low bone turnover condition. Many factors are involved in the complex relationships between bone loss and PH. Since bone loss was mainly reported in patients with fasting hypercalciuria, a primary alteration in bone metabolism was proposed as a cause of both hypercalciuria and bone demineralization. This hypothesis was strengthened by the observation that some bone resorbing-cytokines, such as IL-1, IL-6, and TNF- $\alpha$ are high in hypercalciuric patients. The effect of an excessive response to the acid load induced by dietary protein intake seems an additional factor explaining a primitive alteration of bone. The intestine plays a major role in the clinical course of bone disease in PH. Patients with absorptive hypercalciuria less frequently show bone disease and a reduction in dietary calcium greatly increases the probability of bone loss in $\mathrm{PH}$ subjects. It has recently been reported that greater bone loss is associated with a larger increase in intestinal calcium absorption in PH patients. Considering the absence of PTH alterations, it was proposed that this is not a compensatory phenomenon, but probably the marker of disturbed cell calcium transport, involving both intestinal and bone tissues. While renal hypercalciuria is rather uncommon, the kidney still seems to play a role in the pathogenesis of bone loss of $\mathrm{PH}$ patients, possibly via the effect of mild to moderate urinary phosphate loss with secondary hypophosphatemia. In conclusion, bone loss is very common in $\mathrm{PH}$ patients. Even if most of the factors involved in this process have been identified, many aspects of this intriguing clinical condition remain to be elucidated.

KEY WORDS: bone density, intestinal calcium absorption, osteoporosis, phosphate metabolism, primary hypercalciuria.

\section{Introduction}

Hypercalciuria is a common metabolic defect that can frequently be detected by clinicians in several conditions. This alteration was firstly described by Flocks and colleagues in 1939. However, it was Albright who first introduced the term "Idiopathic (or Primary) Hypercalciuria" to define a form of elevated urinary calcium excretion that can be observed in spite of the absence of any clinical conditions or treatments capable of inducing this defect (1). Nowadays, hypercalciuria is a well-recognized condition, which especially affects patients with kidney stones with an incidence of $50-60 \%$ in these subjects (2-4).

The use of appropriate reference ranges for normal urine calcium excretion is mandatory in order to make a correct diagnosis of hypercalciuria. However, the definition of the upper normal limit for urine calcium excretion is still a matter of debate. Many years ago, Pak et al. (5) defined hypercalciuria as a condition characterized by 24-hour urine calcium excretion greater than $250 \mathrm{mg} /$ day in females and $300 \mathrm{mg} /$ day in males, while patients are on their usual diet. In the attempt to adjust urine calcium excretion for body weight, they also defined hypercalciuria as a 24-hour urine excretion greater than $4 \mathrm{mg} / \mathrm{kg}$ of body weight/day in both sexes (5). This definition was criticized because it was based on a study on an adult population of a particular geographic area that did not provide any corrections for the dietetic calcium intake of the subjects studied. This could limit the appropriateness of this definition, especially in the case of particular classes of persons, such as infants or elderly people. However, very few attempts have been made to solve the issue. Heaney et al. (6) tried to redefine the normal limits for urine calcium excretion by adjusting for menopausal status and dietary calcium intake. They found that in normal postmenopausal women at moderate or high calcium intake 24hour urine calcium excretion exceeded the normal value of 250 $\mathrm{mg} /$ day and they postulated that for this subset of subjects the upper normal limit of calcium excretion should be considered at least $286 \mathrm{mg}$ of calcium per day. However, it is well-known that even in healthy adults dietary calcium intake rarely approaches moderate or high values, being very frequently moderate or frankly below the normal requirements, at least in Western countries (7). In these subjects, the upper limits for 24-hour urine calcium excretion found by Heaney et al. (6) were very close to those reported by Pak et al. (5). Consequently, on the ground of the currently available evidence and in view of future more reliable data, it is reasonable to use Pak et al's definition, which was formulated on the basis of a larger cohort of patients than that of Heaney and colleagues, at least for adult people. In contrast, because of the marked differences in calcium metabolism and dietary habits, it is still rather questionable whether these limits should be adopted tout court for infants and adolescents.

To make a proper diagnosis of hypercalciuria a 24-hour urine collection without dietetic manipulations must be obtained. Considering the importance of the pre-analytical variability, which is mainly related to possible errors in urine collection and conservation, the assessment of urine calcium excretion on two consecutive 24-hour samples is generally recommended to confirm the presence of hypercalciuria. However, problems in 
both urine collection and storage tend to induce under- rather than overestimation of calcium excretion. Thus, in the absence of a true analytical error, it is rather unlikely that high urine calcium excretion may represent a false positive result.

Apart from pathological conditions (Tab. I), several factors influence urinary calcium excretion in humans. Among these, the most important are sex, body weight, and intake of a number of nutrients, such as sodium, potassium, phosphate, proteins, carbohydrates, as well as alcohol. The amount of dietary calcium and the efficiency of intestinal calcium absorption are particularly important. Healthy subjects are estimated to eliminate about $6-7 \%$ of dietary calcium in urine daily. Consequently, depending on the different ages, an excess of dietary calcium (greater than $1500 \mathrm{mg} /$ day) can be associated with the development of hypercalciuria.

Dietary sodium intake may affect calcium excretion by increasing it of about $20-40 \mathrm{mg} /$ day for every increment of $2.3 \mathrm{~g}$ of sodium (8). Phosphate metabolism largely influences urine calcium excretion. Phosphate leak and consequent hypophosphatemia stimulate renal production of $1,25-(\mathrm{OH})_{2}$ vitamin $\mathrm{D}$, which in turn increases intestinal calcium absorption, then possibly leading to hypercalciuria. Several epidemiological studies confirmed the association between dietary protein intake and calcium metabolism and its excretion. Some investigators con-

Table I - Causes of secondary hypercalciuria

Diet-dependent

Excessive dietary intake of

- Calcium

- Sodium

- Animal proteins

- Carbohydrates

- Alcohol

Reduced intake/absorption of

- Phosphate

- Potassium

Secondary increase in intestinal calcium absorption

Vitamin D therapy

Endogenous over production of $1,25-(\mathrm{OH})_{2}$ vitamin $\mathrm{D}$

- Primary hyperparathyroidism

- Granulomatous diseases

- Lymphomas

- Severe hypophosphatemic diseases

Increased osteoclastic resorption of bone

Bone metastases

Multiple myeloma

Primary hyperparathyroidism

Paget's disease of bone

Hyperthyroidism

Prolonged immobility

Reduced renal tubular reabsorption of calcium

Loop diuretics

Bartter syndrome

Medullary sponge kidney disease

Primary renal tubular defects

Endogenous/exogenous glucocorticoid excess

Genetic alterations (chloride channels, calcium-sensing receptor) cluded that dietary proteins are more important as regulators of urinary calcium than dietary calcium intake (9-11). Moderately protein diets (in the range of $1.0-1.5 \mathrm{~g}$ proteins $/ \mathrm{kg}$ ) are associated with normal calcium metabolism and presumably do not alter skeletal homeostasis. Subjects with high protein intake, particularly from omnivorous sources, develop sustained hypercalciuria. This seems to be due to an increase in bone resorption, secondary to the excessive acid load, as well as to the direct effects of proteins on calcium handling at the renal tubular and intestinal level (11).

Hypercalciuria is commonly distinguished in a secondary form when it is associated with conditions that can induce its presence (Tab. I), or primary, in the absence of a specific cause.

\section{Primary Hypercalciuria (PH)}

\section{Definition}

Primary (or Idiopathic) Hypercalciuria is defined as the form of excessive calcium excretion that is not associated with conditions known to increase calcium elimination. This definition, which was first used by Albright (1), was almost completely maintained by Pak et al. (5). However, according to the more recent view that dietetic factors can widely influence urine calcium excretion, "true" primary hypercalciuria is considered the form of urinary calcium excess that does not depend on known conditions and in which possible dietetic disturbances have been eliminated by keeping patients on a daily diet with $1000-1200 \mathrm{mg}$ of calcium and no more than $1-1.5 \mathrm{~g} / \mathrm{kg}$ body weight (BW) of proteins (11). This definition can be considered restrictive as compared to that by $\mathrm{Al}-$ bright and Pak. According to the new definition, some patients are no longer classified as hypercalciuric as before. However, it is well-known that a diet exceeding the above-mentioned range for daily calcium and protein intake affects calcium excretion even in patients with no evidence of intrinsic alterations of calcium metabolism, who would be misclassified as hypercalciuric.

Already in the Eighties, Pak et al. (12) classified PH by dividing it into three distinct pathogenetic pathways, according to three independent primary metabolic defects: 1) absorptive hypercalciuria type I, when primary intestinal hyperabsorption of calcium is involved; 2) absorptive hypercalciuria type III, when a primary renal leak of phosphate is present, thus inducing hypophosphatemia and secondarily $1,25-(\mathrm{OH})_{2}$ vitamin D-mediated intestinal hyperabsorption of calcium; 3 ) renal hypercalciuria, when a primary renal leak of calcium with secondary compensatory hyperparathyroidism is present. Pak et al. also distinguished a form of so-called "reabsorptive hypercalciuria", when hypercalciuria is induced by an excessive output of calcium from bone, for example in patients with Primary Hyperparathyroidism.

However, this classification has widely been revised over the last twenty years on the basis of new pathophysiological observations and of a more thorough clinical and laboratory examination of patients affected by this condition. Nowadays, it is believed that, beyond the need for a classifying approach, three different pathophysiological mechanisms, possibly overlapping and operating together in a complex network, are involved in the pathogenesis of hypercalciuria: increased intestinal absorption of calcium, primary excessive calcium release from bone, and altered renal calcium handling.

\section{Pathophysiology}

1. Increased intestinal absorption of calcium

Recent studies definitely confirmed that calcium absorption is almost invariably increased in patients with $\mathrm{PH}$ (13). Broadus 
and colleagues found that $1,25-(\mathrm{OH})_{2}$ vitamin D metabolism can be altered in patients with absorptive hypercalciuria, then being responsible for the intestinal calcium hyperabsorption seen in these patients (14). Since these observations have not been confirmed by others, it has been proposed that intestinal calcium absorption may be increased irrespective of the elevation in $1,25-(\mathrm{OH})_{2}$ vitamin $\mathrm{D}$. Indeed, in rats with genetic-determined hypercalciuria an increased expression of the vitamin $D$ receptor at the intestinal level was reported (15), thus explaining the reason why intestinal calcium absorption can be increased even in the absence of elevated values of serum $1,25-(\mathrm{OH})_{2}$ vitamin D. However, there is no evidence that a similar mechanism may be operating in humans (16). When present, altered renal handling of phosphate with excessive phosphaturia, which will then induce hypophosphatemia, may be responsible for overproduction of $1,25-(\mathrm{OH})_{2}$ vitamin $\mathrm{D}$ with secondary intestinal calcium hyperabsorption (17).

\section{Renal tubular calcium leak}

A defect in renal tubular calcium reabsorption was demonstrated in both humans and genetic hypercalciuric stone forming rats as a cause of idiopathic hypercalciuria $(18,19)$. However, an extensive revision of the cases of so-called "renal hypercalciuria" demonstrated that, in contrast with previous reports, the two distinctive characteristics of this form - that is, low-normal serum calcium and increased PTH levels - were present in a small minority of patients with $\mathrm{PH}(20)$. It is currently believed that no more than $2-3 \%$ of $\mathrm{PH}$ cases are generated by a true calcium leak.

\section{Fasting Hypercalciuria}

Most patients with $\mathrm{PH}$ show persistence of elevated urine calcium even after a low calcium diet or in fasting conditions, which is not associated with any secondary parathyroid hyperfunction. This metabolic pattern tends to exclude a major role for both intestinal calcium hyperabsorption and renal calcium leak. The term Fasting Hypercalciuria was formulated for this condition and it was assumed that primary excess of calcium output from bone explained this defect (21).

\section{Diagnosis of $\mathrm{PH}$}

Once the diagnosis of hypercalciuria is correctly made, it is necessary to exclude any secondary causes of hypercalciuria (Tab. I). In the presence of Primary Hypercalciuria, it is generally advisable to perform some simple additional laboratory tests to identify the main metabolic defect (Fig. 1). Briefly, after a week of low calcium, normal sodium, and protein dietary intake, a fasting morning urine sample for the determination of calcium and creatinine is obtained from the patient. If urine calcium excretion normalizes, with the ratio of urine calcium/creatinine falling below the value of $0.11 \mathrm{mg} / \mathrm{mg}$, the diagnosis of Absorptive Hypercalciuria can be made. If urine calcium excretion remains elevated in spite of dietary calcium deprivation, the diagnosis of Fasting Hypercalciuria is reasonable. On the basis of serum calcium and PTH values, this form can be further classified as Undetermined Fasting Hypercalciuria or Renal Hypercalciuria.

\section{$\mathrm{PH}$ and bone disease}

\section{The size of the problem}

Since the Seventies, the hypothesis that continuous elevation in urine calcium excretion could be associated with some degree of bone loss has been more clearly defined. Due to the

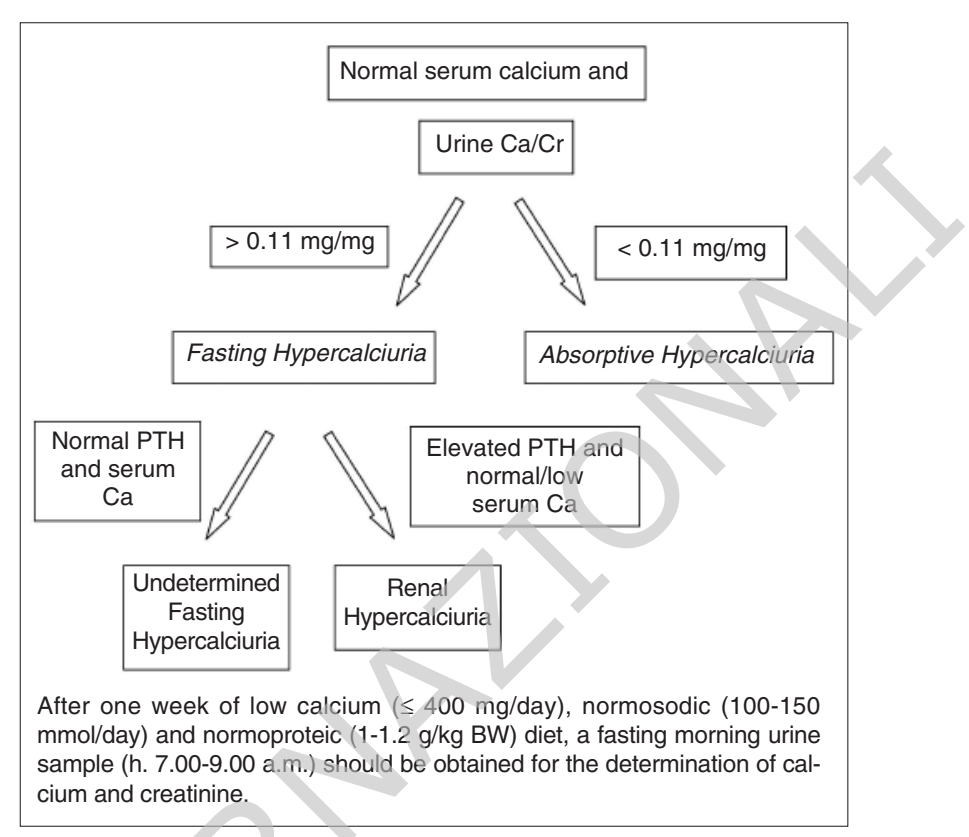

Figure 1 - Flow-chart for the classification of Primary Hypercalciuria.

fact that idiopathic hypercalciuria is one of the most common phenotypes in patients with kidney stones, the large majority of the studies undertaken to assess bone status in hypercalciuric patients were conducted in patient with calcium nephrolithiasis. On the whole, these studies demonstrated that while bone density is substantially normal or only slightly reduced in patients with calcium nephrolithiasis without hypercalciuria, significant bone loss is present in patients with kidney stones and primary hypercalciuria (Tab. II). Bone loss seems to mainly involve those skeletal sites where trabecular bone is more represented, such as vertebral bodies (22-35). However, a reduction in femoral density was reported by several authors $(28,29,31-36)$. There are no data on the number of hypercalciuric patients who suffer from an established osteoporotic bone disease as defined by WHO classification (37). Yet, the rate of demineralization is generally substantial, ranging from 10 to $15 \%$ as compared to age- and sexmatched normal subjects $(24,26-28,31,33)$. Some authors reported even more significant decreases in bone density (25). The importance of these results from a clinical point of view is further strengthened by the relatively young age (approximately 50 years) of these patients and by the large proportion of males in the studied populations.

There are no data on the role of hypercalciuria as a risk factor for fractures. However, an increased fracture risk was reported in patients with renal calculi $(38,39)$. There is no direct evidence in these studies that hypercalciuria per se is responsible for the increased fracture risk in these patients. However, among patients with kidney stones bone loss is a predominant, if not exclusive, characteristic of hypercalciuric subjects. Consequently, it can be speculated that hypercalciuria can be at least one of the factors explaining the increased proportion of fragility fractures seen in these patients.

Besides the wide overlap between the pathophysiology of absorptive and fasting hypercalciuria, the differences in bone involvement in patients with these two forms of hypercalciuria are still matters of debate. While most authors specifically observed a significant proportion of bone loss in patients with fasting hypercalciuria but not in those with the absorptive form $(24-26,31,33)$, others reported a decrease in bone density irrespective of the type of primary hypercalciuria $(27,29,30)$, 
Table II - Bone mineral density (BMD) in patients with primary hypercalciuria.

\begin{tabular}{llll}
\hline Author (reference), year & Measurement method & Measurement site & Result of BMD \\
\hline Lawoyin et al. (22), 1979 & SPA & Radius & $\downarrow$ \\
Fuss et al. (23), 1983 & SPA & Radius & Spine \\
Pacifici et al. (24), 1990 & QCT & Spine & $\downarrow$ \\
Bataille et al. (25), 1991 & QCT & Spine \\
Borghi et al. (26), 1991 & DPA & Spine, radius & $\downarrow$ \\
Pietschmann et al. (27), 1992 & DEXA, SPA & Spine, femur & $\downarrow$ \\
Jaeger et al. (28), 1994 & DEXA & Spine, femur & $\downarrow$ \\
Weisinger et al. (29), 1996 & DEXA & Spine & $\downarrow$ \\
Ghazali et al. (30), 1997 & QCT & Spine, femur & $\downarrow$ \\
Giannini et al. (31), 1998 & DEXA & Spine, femur & $\downarrow$ \\
Misael da Silva et a.l (32), 2002 & DEXA & Spine, femur & $\downarrow$ \\
Tasca et al. (33), 2002 & DEXA & Spine, femur & $\downarrow$ \\
Asplin et al. (34), 2003 & DEXA & Spine, femur & $\downarrow$ \\
Vezzoli et al. (35), 2003 & DEXA & Radius, finger & $\downarrow$ \\
Caudarella et al. (36), 2003 & DEXA, QUS & $\downarrow$ \\
\hline
\end{tabular}

DEXA: Dual Energy X-ray Absorptiometry; DPA: Dual Photon Absorptiometry; QCT: Quantitative Computed Tomography; QUS: Quantitative UltraSound; $S P A$ : Single Photon Absorptiometry; $N$ : normal; $\downarrow$ : reduced.

that is, even in patients classified as having Absorptive Hypercalciuria (27-29). Theoretically, the latter finding could appear rather surprising, if one considers that patients with Absorptive Hypercalciuria should be protected from bone loss by the same mechanism generating hypercalciuria - that is, increased intestinal calcium absorption - with a consequent positive calcium balance. However, as to this point, several aspects have to be considered. For one thing, some of the studies focusing on this specific issue were unfortunately carried out population samples that were on too small, which made it difficult to properly compare the consequences on bone of the two forms of hypercalciuria (29). Secondly, a negative effect on bone of a low calcium diet, inappropriately prescribed by the patients themselves or by the physician in order to reduce intestinal calcium absorption, may in turn induce a decrease in bone density even in patients with Absorptive Hypercalciuria. This was apparently the case of the study by Pietschmann et al. (27), who found that their patients with absorptive hypercalciuria had low bone density, although its prevalence was of limited importance as compared to patients with fasting hypercalciuria. Additionally, they speculated that low bone density might also be a consequence of the excessive stimulation of bone resorption resulting from high consumption of dietary protein, increased serum levels of $1,25-(\mathrm{OH})_{2}$ vitamin $\mathrm{D}$, or sensitivity to this hormone in these patients.

New and more intriguing hypotheses are currently being contemplated to solve this issue. One possible explanation is the fact that the absorptive and fasting types of hypercalciuria may not be two truly different forms of defect, but rather a unique disorder, possibly consisting in a systemic alteration of calcium transport that may present a prevalence of intestinal calcium absorption or bone loss. The major support to this view is a recent study in which it was found that hypercalciuric patients with the largest proportion of bone loss also present the highest levels of intestinal calcium absorption (35).

\section{Bone disease type}

One of the most puzzling aspects of bone disease in patients with $\mathrm{PH}$ is its nature. As a matter of fact, bone histomorphome- tric studies are rare in this setting and have not yielded homogeneous results. Bone resorption activity seems to be either increased (40-42) or normal (43), while the most common histological alteration is a reduction in bone formation function, as observed by most authors (40-45). These results tend to be in contrast with those reported for bone turnover markers. Most authors observed increased levels of both bone formation and resorption markers in hypercalciuric patients $(24-26,31,33$, 46). Additional uncertainness may arise from the observation of a moderate to severe mineralization defect, associated with a prolonged mineralization lag $(40,42,43,45)$. Increased osteoid thickness was also reported by Thomas and coworkers (44). Because of the differences in the study populations (type of $\mathrm{PH}$, sex, age, dietetic conditions, and so on) these findings cannot be univocally interpreted. However, taken as a whole, these data seem to refer to a type of skeletal alteration ranging from a moderately low-turnover osteoporosis to an osteomalacic trait.

\section{Pathophysiology}

Whatever the pathophysiological mechanism, the fact that primary hypercalciuria and bone diseases are strictly linked is well-established on the ground of several direct and indirect observations. The rate of urine calcium excretion was found to correlate with bone loss $(29,35,47)$ and elevation in bone turnover markers in hypercalciuric patients $(25,31$, $33,46)$. In addition, several retrospective and prospective studies showed that thiazide use is associated with a reduction in fracture incidence (48-53) and an increase in bone density (54-56). Although thiazides may act directly on bone resorption $(55,56)$, the reduction in renal calcium excretion remains the most important contributing factor to the improvement in bone density detected in thiazide-treated subjects (54-56).

To understand the relationships between $\mathrm{PH}$ and bone loss and the pathogenetic factors shared by the two conditions is even more difficult. For this review, we will briefly examine the role of bone, kidney, and intestine in the pathogenesis of skeletal alterations of $\mathrm{PH}$. 


\section{Bone}

Since the revision of the types of $\mathrm{PH}$ proposed by Levy et al. (20), the term "fasting hypercalciuria" has been used to identify patients who cannot lower or normalize their urine calcium excretion appropriately after a restriction in dietary calcium consumption. As low bone density was more frequently reported in these patients, the presence of some conditions causing simultaneously hypercalciuria and bone demineralization was suggested.

Pacifici et al. (24) firstly reported that some cytokines contributing to the mechanisms that regulate bone resorption may be involved in the pathogenesis of bone in patients with $\mathrm{PH}$. They found that monocytes in patients with fasting hypercalciuria, but not in those with the absorptive form, produced an exaggerated amount of interleukin-1 (IL-1), a well-known very potent stimulator of bone resorption processes (57), which in turn was correlated with a significant degree of bone demineralization. The role of cytokines in this setting was then confirmed by other reports. Weisinger and coworkers (29) found that the production and mRNA expression of IL-1 $\alpha$ from unstimulated peripheral blood mononuclear cells correlated with spinal bone loss in patients with $\mathrm{PH}$ and nephrolithiasis. In addition, the same cells produced an increased amount of IL- $1 \alpha$, interleukin6 (IL-6), and Tumoral Nechrosis Factor- $\alpha$ (TNF- $\alpha$ ) as compared to controls after stimulation with lipopolysaccharide (LPS). Since all these cytokines are considered local mediators of bone resorption (58), the authors concluded that bone loss may largely depend upon these alterations in hypercalciuric patients with calcium stones. Similar results were obtained by Ghazali et al. (30), who found that IL-1 $\beta$, IL-6, TNF- $\alpha$, and Granulocyte Macrophage-Colony Stimulating Factor (GM-CSF) from peripheral blood monocytes were involved in the pathogenesis of bone loss in patients with $\mathrm{PH}$. The consistency of all these results undoubtedly strengthens the importance of cytokines as pathogenetic factors of bone loss in PH (Tab. III). However, it remains to be elucidated whether overproduction of these cytokines from bone and bone marrow cells is also present. Indeed, even if it is believed that altered cytokine secretion from peripheral mononuclear cells may in some way reflect a similar pattern in bone marrow (59), all these bone-reabsorbing substances are mainly considered local regulating factors of cell differentiation and function (58). In addition, no clear explanations were given for such an alteration in cytokine secretion in patients with $\mathrm{PH}$ and no differences in IL-1 $\beta$ gene polymorphism were found between patients with or without $\mathrm{PH}$ (60).

Other factors are thought to be involved in bone alteration in $\mathrm{PH}$. One of the most studied features is the effect of protein intake in these patients. Excessive protein intake, especially of animal origin, was found to sharply increase urine calcium ex- cretion and bone resorption and lead to bone loss (11). The main responsible mechanism for these effects is the acid load produced by proteins, especially by those rich in sulfur-containing amino acids (11). Accordingly, it was demonstrated that sulfate excretion and some markers of protein intake, such as urinary or serum urea, well correlate with bone turnover markers and density $(25,27,28,61)$. We also found that a moderate protein restriction was accompanied by a proportional reduction in calcium excretion and bone turnover markers in patients with nephrolithiasis and $\mathrm{PH}(61)$. Since dietary protein excess was repeatedly reported in hypercalciuric stone formers $(11,61)$ and hypersensitivity to protein effects on bone was also suggested, normalization of protein intake is highly recommended in hypercalciuric patients.

No consistent data currently support the substantial role of calciotropic hormones in the pathogenesis of bone loss in $\mathrm{PH}$. $1,25-(\mathrm{OH})_{2}$ vitamin $\mathrm{D}$ was reported to be higher in $\mathrm{PH}$ patients than in controls and it was observed that this hormone can induce an increase in bone resorption (62). However, the elevation in $1,25-(\mathrm{OH})_{2}$ vitamin $D$ levels was more frequently described in patients with absorptive hypercalciuria, whose bone density levels are generally normal or poorly diminished. Bataille et al. (25) also found that $1,25-(\mathrm{OH})_{2}$ vitamin D levels have a protective rather than a damaging effect on bone mass in patients with $\mathrm{PH}$ and kidney stones. Apart from the very small proportion of patients that can be classified as having renal hypercalciuria (20), PTH levels are generally normal in $\mathrm{PH}$ patients and are not thought to have a significant role in the pathogenesis of bone loss in this setting.

\section{Intestine}

Although the classical distinction of $\mathrm{PH}$ in absorptive and fasting hypercalciuria is still maintained, a wide overlap seems to occur between the two forms. Besides, the intestinal function plays a key role both in the pathogenesis of $\mathrm{PH}$ and in the development or maintenance of bone disease. As mentioned above, some studies also reported a decrease in bone density in patients with absorptive hypercalciuria (27). On the other hand, increased intestinal calcium absorption is frequently present even in patients with fasting hypercalciuria (19). Vezzoli and coworkers recently reconsidered the complex relationships between intestine and bone in patients with $\mathrm{PH}$ in a very interesting study (35). They assessed intestinal calcium absorption in hypercalciuric patients through the stable strontium method. They found that the greater the loss of bone mineral density, the larger the increase in intestinal calcium absorption, the latter being the best predictor of bone mass in a multiple regression model (Fig. 2). Since PTH values were similar in hypercalciuric and normocalciuric stone formers, they speculated that this is not a compensatory phenomenon, but probably the

Table III - Cytokines levels from peripheral blood mononuclear cells in patients with $\mathrm{PH}$ and controls.

\begin{tabular}{llllllll}
\hline Author & Population sample & IL-1 & IL-1 $\alpha$ & IL-1 $\beta$ & IL-6 & TNF- $\alpha$ & GM-CSF \\
\hline Pacifici et al. (24) & FH, AH, Controls & $261 \pm 81^{\text {a }}$ & - & - & - & - & - \\
Weisinger et al. (29) & $\mathrm{IH}, \mathrm{NC}$, Controls & - & $680 \pm 139^{\mathrm{b}}$ & - & $19 \pm 4^{\mathrm{c}}$ & $2976 \pm 417^{\mathrm{b}}$ & - \\
Ghazali et al. (30) & $\mathrm{IH}, \mathrm{DH}$, Controls & - & - & $40 \pm 21^{\mathrm{d}}$ & $347 \pm 145^{\mathrm{e}}$ & $236 \pm 136$ & $52 \pm 27^{\mathrm{e}}$ \\
\hline
\end{tabular}

FH = Fasting Hypercalciuria; $\mathrm{AH}=$ Absorptive Hypercalciuria; $\mathrm{IH}=$ Idiopathic Hypercalciuria; NC = Normocalciuria; $\mathrm{DH}=$ Dietary Hypercalciuria; $\mathrm{LPS}=$ Lipopolysaccharide

${ }^{a} \mathrm{FH} \mathrm{P}<0.01$ vs $\mathrm{AH}$ and Controls

${ }^{b}$ IH $P<0.01$ vs NC and Controls (LPS-stimulated)

${ }^{c} \mathrm{IH} \quad \mathrm{P}<0.05$ vs NC and Controls (LPS-stimulated)

${ }^{d} \mathrm{IH} P<0.01$ vs DH

${ }^{\mathrm{e}} \mathrm{IH} \mathrm{P}<0.05$ vs Controls. 


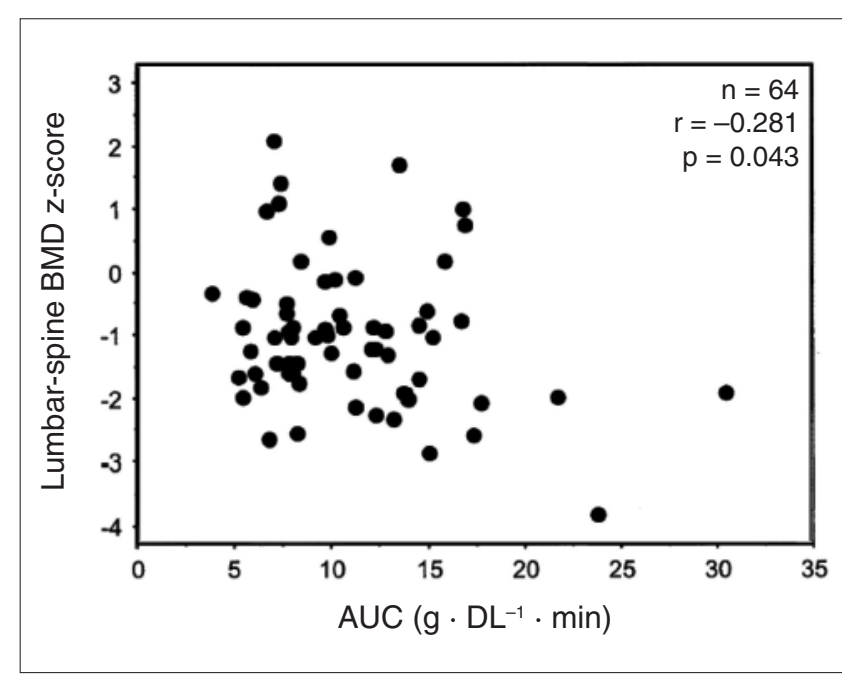

Figure 2 - Multiple stepwise regression analysis showing that lumbar-spine BMD z-score was negatively associated with enteral strontium absorption (AUC, $x$ axis) in hypercalciuric stone-forming women (reproduced with permission by Vezzoli et al., ref. 35).

marker of disturbed cell calcium transport, involving both intestinal and bone tissues (35). This hypothesis would also be in keeping with the view that absorptive and fasting hypercalciuria may be different phenotypes and expressions of the same disorder (21). Although its nature is not fully understood, some genetic influences might be possible (63).

Whatever the explanation for the increased intestinal calcium absorption in patients with $\mathrm{PH}$, the importance of this observation is further strengthened by the fact that, in the absence of proportional intestinal calcium hyperabsorption, the negative calcium balance observed in patients with fasting hypercalciuria should be much larger than it actually is, with a tremendous impact in terms of bone loss and fracture risk. Even if intestinal calcium absorption varies according to dietary calcium intake, food quality, intestinal function, serum $1,25-(\mathrm{OH})_{2}$ vitamin $\mathrm{D}$, and so forth, approximately $4-5 \mathrm{mmol}$ of calcium are absorbed daily through the gut and the same amount is eliminated with the urines (Fig. 3, ref. 63). In the presence of hypercalciuria, calcium balance can be maintained only at the expenses of the skeletal tissue or through an increase in intestinal calcium absorption, which can in turn limit bone loss. The restriction in dietary calcium intake, which many hypercalciuric patients tend to do by themselves or after medical prescription, is therefore a major risk factor for bone loss in this setting. Indeed, it was clearly seen that a reduction in calcium intake is associated with negative calcium balance and bone loss in hypercalciuric patients $(28,64,65)$. Some authors $(64,65)$ reported this negative effect after a calcium-restricted diet of 2-8 years, while Jaeger et al. observed a significant reduction in bone density in hypercalciuric patients already after the first year of low calcium diet (9). In addition, Curhan et al. (66) reported that dietary calcium restriction does not reduce the incidence of new kidney stones but, in fact, it increases the risk of developing new symptomatic renal calculi, at least in males. This seems to occur because of the increase in intestinal oxalate absorption with a secondary increase in urinary excretion in the absence of calcium in the colon. All these observations suggest that hypercalciuric patients need to maintain an appropriate dietary calcium intake.

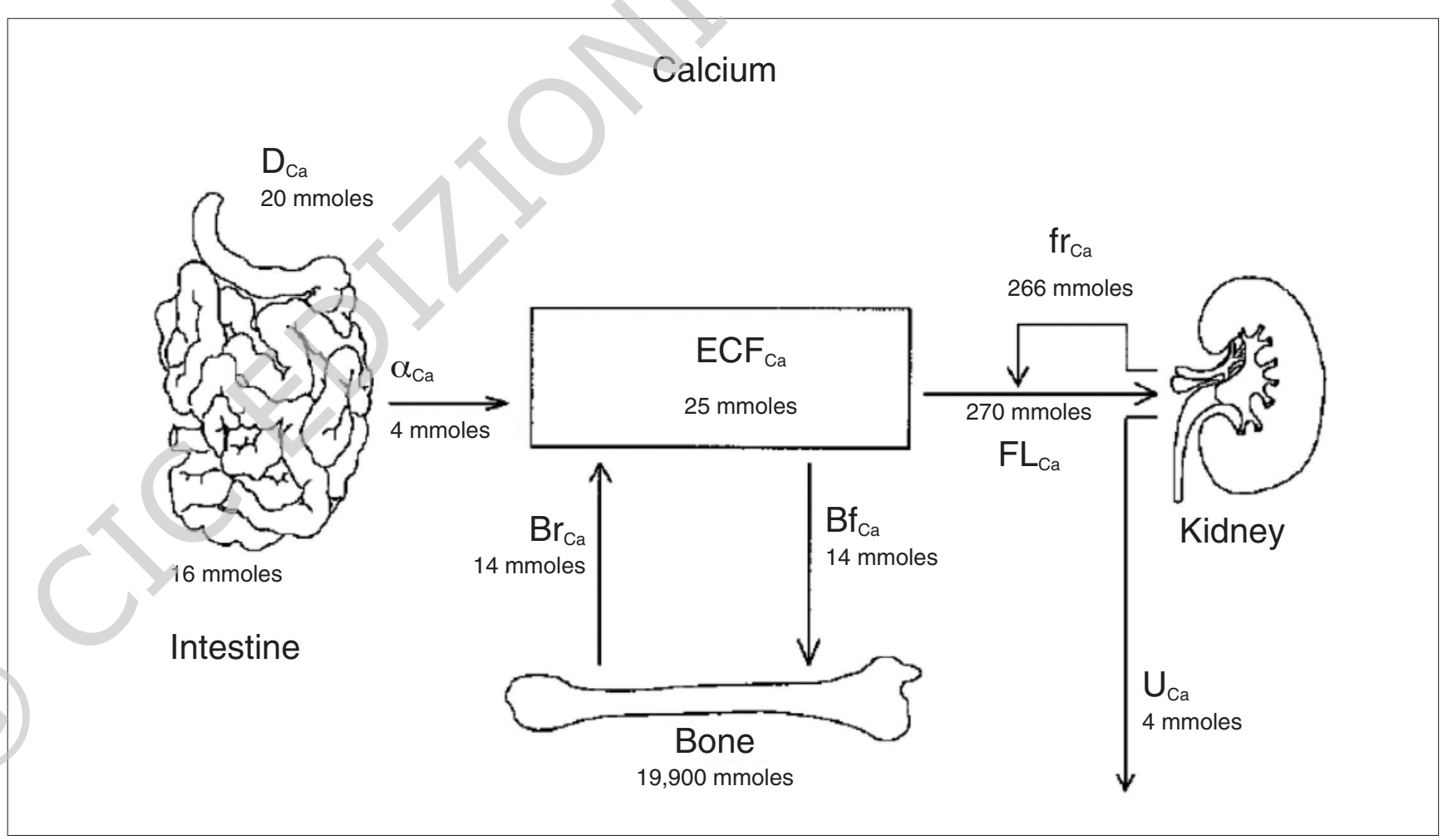

Figure 3 - Anatomic sites of calcium flux. The figure shows the major sites of calcium flux in the body with indication of the average amounts of daily calcium flux. Dietary (D) calcium (Ca) undergoes net absorption (a) through the intestine into the extracellular fluid (ECF). Daily bone resorption (Br) and formation $(\mathrm{Bf})$ are equal. The filtered load (FL) of calcium is substantially reabsorbed (fr, fractional reabsorption) in the kidney, resulting in urine (U) calcium excretion that is virtually identical to the amount absorbed in the intestine (reproduced with permission from Frick and Bushinsky, ref. 63). 


\section{Kidney}

The presence of renal calcium leak is the basis for the socalled renal hypercalciuria, which is characterized by increased urine calcium, a tendency toward hypocalcemia, and a secondary increase in parathyroid hormone secretion that is considered the main cause of bone loss in these patients (64). However, the large revision of the pathogenetic aspects of patients with $\mathrm{PH}$ led to the observation that less than $5 \%$ of hypercalciuric patients suffer from a renal form of $\mathrm{PH}$ (20). As a consequence, the importance of renal calcium leak as a pathogenetic factor for bone loss in these patients was completely reconsidered.

However, some other aspects seem to link the kidney to the complex relationships occurring between hypercalciuria and bone. Increased urinary phosphate excretion was found in hypercalciuric patients as compared to normal subjects, irrespective of the presence of a true form of absorptive hypercalciuria with renal calcium leak (67). It was suggested that excessive excretion of phosphate may be present in the majority of patients with $\mathrm{PH}$, then concurring to the development of the hypercalciuric state in the whole population of $\mathrm{PH}$ patients. Similar findings were reported by Prié et al. (68) in hypercalciuric stone formers. They observed that the distribution of renal phosphate threshold normalized for glomerular filtration rate (TmPi) was quite different between patients and controls, with hypercalciuric patients showing a decreased value of $\mathrm{TmPi}$ in approximately $20 \%$ of cases. No assessment of bone status was made in the two papers. However, it could be hypothesized that the alteration in phosphate metabolism seen in these patients plays a role also in the pathogenesis of bone damage in hypercalciuric patients. Hereditary hypophosphatemic rickets with hypercalciuria $(\mathrm{HHRH})$ is a paradigm of this pathophysiology (69). This disease shares some clinical aspects with the disorder seen in the Npt2 knockout mice, carrying the deletion of the gene of kidney-specific Na-Pi cotransporter, in which a delay in bone mineralization is seen 21 days after birth. These bone alterations may resemble those observed in hypercalciuric patients in histomorphometric studies (40-45), in which an alteration in the bone mineralization process was reported. Accordingly, a mutation of NPT2 gene was found in nephrolithiasic patients with decreased bone density by Prie and coworkers (70). In conclusion, even if no clear evidence supports the hypothesis that renal phosphate leak may be, at least in part, responsible for bone disease in $\mathrm{PH}$ patients, this research field appears as one of the most promising to better elucidate the role of kidney in the pathogenesis of bone loss in $\mathrm{PH}$.

\section{Primary Hypercalciuria and Osteoporosis}

Despite the evidence that Primary Hypercalciura is very frequently associated with bone loss and the fact that 24-hour urine collection for calcium assessment is recommended by the large majority of the international guidelines on the diagnostic process for osteoporotic patients, no systematic attempt has been made to ascertain the role of this defect in patients

Table IV - Prevalence of PH in otherwise healthy osteoporotic patients.

\begin{tabular}{lcc}
\hline Author & Year & Prevalence of PH \\
\hline Tannenbaum et al. (71) & 2002 & $9.8 \%$ \\
Deutschmann et al. (72) & 2002 & $10.3 \%$ \\
Peris et al. (73) & 2002 & $21 \%$ \\
Giannini et al. (74) & 2003 & $19 \%$ \\
\hline
\end{tabular}

with osteoporosis up to the last two years. Tannenbaum and colleagues (71) first reported prevalence of Primary Hypercalciuria of about $10 \%$ in a large sample of otherwise healthy women with osteoporosis. In 2002, Deutschmann et al. found that idiopathic hypercalciura was present in about $10 \%$ of their patients with apparent primary osteoporosis, with a frequency of $7.7 \%$ in females and $20.7 \%$ in males (72). In the same year, Peris et al. described prevalence of hypercalciuria of about $20 \%$ in premenopausal women with osteoporosis, but without any signs or symptoms of associated diseases. (73). These studies are very important because they clearly point out the fact that a very large proportion of patients with otherwise primary osteoporosis may have an idiopathic form of hypercalciuria. However, no specific attempt was made by these authors to correlate the presence of hypercalciuria with bone loss. We retrospectively analyzed data of 241 otherwise healthy women referred to our Unit because of osteoporosis and reported the presence of Primary Hypercalciuria in $19 \%$ of the subjects. When these patients were compared with osteoporotic subjects without hypercalciuria, the only differences detected between the two groups apart from urine calcium were urinary phosphate and renal tubular phosphate reabsorption (74). However, the level of hypercalciuria was the most important predictor of bone loss only in patients carrying this defect, with calciuria accounting for more than $50 \%$ of the variance in spinal bone density. Noteworthy is that the levels of renal tubular reabsorption of phosphate were strongly associated with the probable presence of hypercalciuria (OR 0,33, 95\% C.I. 0,18-0,62). We interpreted these data as a further demonstration of the close relationship between hypercalciuria and bone loss, even in patients with an osteoporotic phenotype rather than with kidney stones. This further stresses the need for a proper evaluation of calcium metabolism in osteoporotic patients and for a thorough analysis of the complex pathophysiological relationship between bone disease and hypercalciuria so as to transfer these new findings into a more appropriate and specific therapeutic option.

On the basis of the above considerations, the pathogenesis of the bone disease affecting subjects with both osteoporosis and hypercalciuria may not differ from that of patients with kidney stones, reduced bone mass, and primary hypercalciuria. However, some other mechanisms may contribute to an increase in urine calcium in postmenopausal women with osteoporosis. Heshmati et al. (75) found that postmenopausal women with osteoporosis had a defect in renal calcium conservation as compared to subjects with normal bone density matched for sex and menopausal status. This effect was not dependent upon PTH. Since estrogens can exert their actions even on renal tubules, the authors concluded that estrogen responsiveness may be altered in postmenopausal osteoporotic women, then inducing both an increased skeletal calcium outflow and renal calcium losses. Even if this mechanism is likely to occur in women with osteoporosis and primary hypercalciuria too, there is no direct evidence that can confirm this intriguing hypothesis in this subset of patients.

\section{Conclusions}

Primary Hypercalciuria is a very common finding in patients with kidney stones and otherwise primary osteoporosis. It is now clear that the association between bone loss and $\mathrm{PH}$ does not follow a chance pattern, but it is characterized by a very close relationship. However, the mechanisms involved in the pathogenesis of bone damage in hypercalciuric patients still remain only partially understood. On the other hand, most of the organs and tissues normally involved in the control of calcium and phosphate metabolism seem to take an active part in the 
pathogenesis of the skeletal alterations in patients with $\mathrm{PH}$. The skeletal tissue per se, kidney, and intestine seem to be responsible for the appearance, maintainance, and clinical course of bone loss in hypercalciuric patients, and to operate together in a pattern of multi-tissue metabolic disorder. The adoption and sharing of common laboratory criteria worldwide to promptly detect the presence of $\mathrm{PH}$ will improve the knowledge in this field, which will allow a more successful clinical and therapeutic approach in this very complex and intriguing matter.

\section{References}

1. Abright $\mathrm{F}$, Henneman $\mathrm{P}$, Benedict $\mathrm{PH}$, et al. Idiopathic hypercalciuria: a preliminary report. Proc R Soc Med. 1953;46:1077-1081.

2. Hodgkinson A, Pyrah LN. The urinary excretion of calcium and inorganic phosphate in 344 patients with calcium stone of renal origin. Br J Surg. 1958;46:10-18.

3. Smith LH, VanDenBerg CJ, Wilson DM. Nutrition and urolithiasis. N Engl J Med. 1980;98:87-89.

4. Pak CY. Medical management of nephrolithiasis. J Urol. 1982;128:1157-1164.

5. Pak CY, Ohata M, Lawrence EC, et al. The hypercalciurias: causes, parathyroid functions, and diagnostic criteria. J Clin Invest. 1974;54:387-400.

6. Heaney RP, Recker RR, Ryan RA. Urinary calcium in perimenopausal women: normative values. Osteoporos Int. 1999;9: 13-18.

7. Kerstetter JE, O'Brian KO, Insogna KL. Low protein intake: the impact on calcium and bone homeostasis in humans. J Nutr. 2003; 133:855S-861S

8. Nordin BE, Need AG, Morris HA, et al. The nature and significance of the relationship between urinary sodium and urinary calcium in women. J Nutr. 1993;123:1615-1622.

9. Hegsted M, Schuette SA, Zemel MB, et al. Urinary calcium and calcium balance in young men as affected by level of protein and phosphorus intake. J Nutr. 1981;111:553-562.

10. Zemel MB. Calcium utilization: effect of varying level and source of dietary protein. Am J Clin Nutr. 1988;48:880-883.

11. Kerstetter JE, O’Brien KO, Insogna KL. Dietary protein, calcium metabolism, and skeletal homeostasis revisited. Am J Clin Nutr. 2003:78:584S-592S.

12. Pak CY, Britton F, Peterson R, et al. Ambulatory evaluation of nephrolithiasis. Classification, clinical presentation and diagnostic criteria. Am J Med. 1980;69:19-30.

13. Vezzoli $G$, Tanini A, Ferrucci $L$, et al. Influence of calcium-sensing receptor gene on urinary calcium excretion in stone-forming patients. J Am Soc Nephrol. 2002;13:2517-2523.

14. Broadus $A E$, Insogna $K L$, Lang $R$, et al. Evidence for disordered control of 1,25 -dihydroxyvitamin $D$ production in absorptive hypercalciuria. N Engl J Med. 1984;311:73-80.

15. Li XQ, Tembe V, Horwitz GM, et al. Increased intestinal vitamin D receptor in genetic hypercalciuric rats. A cause of intestinal calcium hyperabsorption. J Clin Invest. 1993:91:661-667.

16. Zerwekh JE, Reed BY, Heller HJ, et al. Normal vitamin D receptor concentration and responsiveness to 1,25-dihydroxyvitamin D3 in skin fibroblasts from patients with absorptive hypercalciuria. Miner Electrolyte Metab. 1998;24:307-313.

17. Prié D, Ravery V, Boccon-Gibod L, et al. Frequency of renal phosphate leak among patients with calcium nephrolithiasis. Kidney Int 2001;60:272-276.

18. Bushinsky DA. Genetic hypercalciuric stone-forming rats. Curr Opin Nephrol Hypertens. 1999;8:479-88.

19. Bataille P, Fardellone $\mathrm{P}$, Ghazali $\mathrm{A}$, et al. Pathophysiology and treatment of idiopathic hypercalciuria. Curr Opin Rheumatol. 1998 10:373-388

20. Levy FL, Adams-Huet B, Pak CYC. Ambulatory evaluation of nephrolithiasis: an update of a 1980 protocol. Am J Med. 1998; 98:50-59.
21. Weisinger JR. New insights in the pathogenesis of idiopathic hypercalciuria; the role of bone. Kidney Int. 1996;49:1507-1518.

22. Lawoyin S, Sismilich S, Browne R, et al. Bone mineral content in patients with calcium urolithiasis. Metabolism. 1979;28:1250-1254.

23. Fuss M, Gillet $\mathrm{C}$, Simon J, et al. Bone mineral content in idiopathic renal stone disease and in primary hyperparathyroidism. Eur Urol. 1983;9:32-34.

24. Pacifici $R$, Rothstein $M$, Rifas $L$, et al. Increased monocyte interleukin-1 activity and decreased vertebral bone density in patients with fasting idiopathic hypercalciuria. J Clin Endocrinol Metab. 1990;71:138-145.

25. Bataille P, Achard JM, Fournier A, et al. Diet, vitamin D and vertebral mineral density in hypercalciuric calcium stone formers. Kidney Int. 1991;39:1193-1205.

26. Borghi L, Meschi T, Guerra A, et al. Vertebral mineral content in diet-dependent and diet-independent hypercalciuria. J Urol. 1991;146:1334-1338.

27. Pietschmann F, Breslau NA, Pak CY. Reduced vertebral bone density in hypercalciuric nephrolithiasis. J Bone Miner Res. 1992;12:1383-1388

28. Jaeger $\mathrm{P}$, Lippuner $\mathrm{K}, \mathrm{Casez} \mathrm{JP}$, et al. Low bone mass in idiopathic renal stone formers: magnitude and significance. J Bone Miner Res. 1994;10:1525-1532.

29. Weisinger JR, Alonzo E, Bellorin-Font E, et al. Possible role of cytokines on the bone mineral loss in idiopathic hypercalciuria. Kidney Int. 1996;49:244-250.

30. Ghazali A, Fuentes V, Desaint C, et al. Low bone mineral density and peripheral blood monocyte activation profile in calcium stone formers with idiopathic hypercalciuria. J Clin Endocrinol Metab. 1997;82:32-38

31. Giannini S, Nobile M, Sartori L, et al. Bone density and skeletal metabolism are altered in idiopathic hypercalciuria. Clinical Nephrology. 1998;50:94-100.

32. Misael da Silva AM, dos Reis LM, Pereira RC, et al. Bone involvement in idiopathic hypercalciuria. Clin Nephrol. 2002;57:183-191.

33. Tasca A, Cacciola A, Ferrarese $P$, et al. Bone alterations in patients with idiopathic hypercalciuria and calcium nephrolithiasis. Urology. 2002;59:865-869.

34. Asplin JR, Bauer KA, Kinder J, et al. Bone mineral density and urine calcium excretion among subjects with and without nephrolithiasis. Kidney Int. 2003;63:662-669.

35. Vezzoli G, Rubinacci A, Bianchin C, et al. Intestinal calcium absorption is associated with bone mass in stone-forming women with idiopathic hypercalciuria. Am J Kidney Dis. 2003;42:1177-1183.

36. Caudarella R, Vescini F, Buffa A. Bone mass loss in calcium stone disease: focus on hypercalciuria and metabolic factors. J Nephrol. 2003;16:260-266

37. Kanis JA. Assessment of fracture risk and its application to screening for postmenopausal osteoporosis: synopsis of a WHO report. WHO Study Group. Osteoporos Int. 1994;4:368-381.

38. Melton LJ III, Crowson CS, Khosla S, et al. Fracture risk among patients with urolithiasis: a population-based cohort study. Kidney Int. 1998,53:459-464.

39. Lauderdale DS, Thisted RA, Wen M, et al. Bone Mineral Density and fracture among prevalent kidney stone cases in the Third $\mathrm{Na}$ tional Health and Nutrition Examination Survey. J Bone Miner Res. 2001;16:1893-1898

40. Heilberg IP, Martini LA, Szejnfeld VL. Bone disease in calcium stone forming patients. Clin Nephrol. 1994;42:175-182.

41. Bordier P, Rychewart A, Gueris J, et al. On the pathogenesis of so-called idiopathic hypercalciuria. Am J Med.1977;63:398-409.

42. Steiniche T, Mosekilde L, Christensen MS, et al. A histomorphometric determination of iliac bone remodeling in patients with recurrent renal stone formation and idiopathic hypercalciuria. Acta Pathol Microbiol Immunol Scand. 1989;97:309-316.

43. Malluche HH, Tschoepe W, Ritz E, et al. Abnormal bone histology in idiopathic hypercalciuria. J Clin Endocrinol Metab. 1980;50:654-658.

44. Thomas J, Roujean J, Aboulker P. Les lésions osseuses dans la lithiase rénale. Leur étude par examen histologique d'un fragment costal. Presse Med. 1962;70: 2437-2440.

45. Zerwekh JE, Sakhaee K, Breslau NA, et al. Impaired bone formation 
in male idiopathic osteoporosis: further reduction in the presence of concomitant hypercalciuria. Osteoporos Int. 1992;2:128-134.

46. Satton RAL, Walker VR. Bone resorption and hypercalciuria in calcium stone formers. Metabolism. 1986;35:485-488.

47. Barkin J, Wilson DR, Manuel MA, et al. Bone mineral content in idiopathic calcium nephrolithiasis. Miner Electrolyte Metab. 1985;11:19-24.

48. Feskanich D, Willett WC, Stampfer MJ, et al. A prospective study of thiazide use and fractures in women. Osteoporos Int. 1997; 7:79-84.

49. LaCroix AZ, Wienpahl J, White LR, et al. Thiazide diuretic agents and the incidence of hip fracture. New Engl J Med. 1990;322:286290.

50. Nguyen TV, Eisman JA, Kelly PJ, et al. Risk factors for osteoporotic fractures in elderly men. Am J Epidemiol. 1996;144:255-263.

51. Cauley JA, Cummings SR, Seeley DG, et al. Effects of thiazide diuretic therapy on bone mass, fractures and falls. The Study of Osteoporotic Fractures Research Group. Ann Intern Med. 1993;118: 666-673.

52. Felson DT, Sloutskis D, Anderson JJ, et al. Thiazide diuretics and the risk of hip fracture. Results from the Framingham Study. JAMA. 1991;265:370-373.

53. Schoofs MWJC, van der Klift M, Hofman A, et al. Thiazide diuretics and the risk for hip fracture. Ann Intern Med. 2003;16:476-482.

54. Adams JS, Song CF, Kantorovich V. Rapid recovery of bone mass in hypercalciuric, osteoporotic man treated with hydrochlorothiazide. Ann Intern Med. 1999;130:658-660.

55. Reid IR, Ames RW, Orr-Walker BJ, et al. Hydrochlorothiazide reduces loss of cortical bone in normal postmenopausal women: a randomized controlled trial. Am J Med. 2000;109:362-370.

56. LaCroix AZ, Ott SM, Ichikawa L, et al. Low-dose hydrochlorothiazide and preservation of bone mineral density in older adults. A randomized, double-blind, placebo-controlled trial. Ann Intern Med. 2000;133:516-526.

57. Gowen M, Mundy GR. Actions of recombinant interleukin 1, interleukin 2 and interferon-gamma on bone resorption in vitro. J Immunol. 1986;136:2478-2482.

58. Manolagas SC. Birth and death of bone cells: basic regulatory mechanisms and implications for the pathogenesis and treatment of osteoporosis. Endocr Rev. 2000;21:115-137.

59. Cohen-Solal ME, Graulet AM, Gueris J, et al. Bone resorption at the femoral neck is dependent on local factors in non osteoporotic late postmenopausal women: an in vivo-in vitro study. J Bone Miner Res. 1995;10:307-315.

60. Chen WC, Wu HC, Chen HY, et al. Interleukin-1beta gene and receptor antagonist gene polymorphisms in patients with calcium oxalate stones. Urol Res. 2001;29: 321-324.
61. Giannini S, Nobile M, Sartori L, et al. Acute effects of moderate dietary protein restriction in patients with idiopathic hypercalciuria and calcium nephrolithiasis. Am J Clin Nutr. 1999;9:267-271.

62. Maierhofer WJ, Gray RW, Cheung HS, et al. Bone resorption stimulated by elevated serum 1,25 $(\mathrm{OH}) 2$ - vitamin D concentrations in healthy men. Kidney Int. 1983;24: 555-560.

63. Frick KK, Bushinsky DA. Molecular mechanisms of primary hypercalciuria. J Am Soc Nephrol. 2003;14:1082-1095.

64. Fuss $M$, Pepersack $T$, Bergman $P$, et al. Low calcium diet in idiopathic urolithiasis: a risk factor for osteopenia as great as in primary hyperparathyroidism. Br J Urol. 1990;65:560-563.

65. Hess B. Low calcium diet in hypercalciuric calcium nephrolithiasis: first do not harm. Scanning Microsc. 1996;10:547-554.

66. Curhan GC, Willett WC, Rimm EB, et al. A prospective study of dietary calcium and other nutrients and the risk of symptomatic kidney stones. N Engl J Med. 1993;328:833-838.

67. Williams $\mathrm{CP}$, Child DF, Hudson PR, et al. Inappropriate phosphate excretion in idiopathic hypercalciuria: the key to a common cause and future treatment? J Clin Pathol. 1996;49:881-888.

68. Prié D, Ravery V, Boccon-Gibod L, et al. Frequency of renal phosphate leak among patients with calcium nephrolithiasis. Kidney Int. 2001;60:272-276.

69. Tieder M, Modai D, Shaked U, et al. "Idiopathic" hypercalciuria and hereditary hypophosphatemic rickets. Two phenotypical expressions of a common genetic defect. New Engl J Med. 1987; 316:125-129

70. Prié D, Huart V, Bakouh N, et al. Nephrolithiasis and osteoporosis associated with hypophosphatemia caused by mutations in the type $2 \mathrm{a}$ sodium-phosphate cotransporter. New Engl J Med. 2002;347:983-991.

71. Tannenbaum C, Clark J, Schwartzman K, et al. Yield of laboratory testing to identify secondary contributors to osteoporosis in otherwise healthy women. J Clin Endocrinol Metab. 2002;87:44314437.

72. Deutschmann HA, Weger M, Weger W, et al. Search for occult secondary osteoporosis: impact of identified possible risk factors on bone mineral density. J Intern Med. 2002;252:389-397.

73. Peris P, Guanabens N, Martinez de Osaba MJ, et al. Clinical characteristics and etiologic factors of premenopausal osteoporosis in a group of Spanish women. Semin Arthritis Rheum. 2002;32:6470.

74. Giannini S, Nobile M, Dalle Carbonare L, et al. Hypercalciuria is a common and important finding in postmenopausal women with osteoporosis. Eur J Endocrinol. 2003;149:209-213.

75. Heshmati HM, Khosla S, Burritt MF, et al. A defect in renal calcium conservation may contribute to the pathogenesis of postmenopausal osteoporosis. J Clin Endocrinol Metab. 1998;83: 1916-1920. 